\section{Case Reports in Ophthalmology}

\title{
A Case of Proliferative Diabetic Retinopathy with HIV Infection in Which HAART Possibly Influenced the Prognosis of Visual Function
}

\author{
Takakuni Kitagaki Takaki Sato Junko Hirai Daisaku Kimura \\ Keigo Kakurai Masanori Fukumoto Kensuke Tajiri \\ Takatoshi Kobayashi Teruyo Kida Shota Kojima Tsunehiko Ikeda \\ Department of Ophthalmology, Osaka Medical College, Takatsuki, Japan
}

\section{Keywords}

Human immunodeficiency virus · HIV · Proliferative diabetic retinopathy · PDR - Vitrectomy . Highly active anti-retroviral therapy $\cdot$ HAART

\begin{abstract}
Background: We report on a patient with proliferative diabetic retinopathy (PDR) and human immunodeficiency virus (HIV) infection who exhibited extremely active PDR followed by a rapid onset of blindness in the right eye. The progression of visual disturbance in the patient's left eye was slowed after starting highly active anti-retroviral therapy (HAART), and vision in that eye was rescued after vitrectomy. Case Report: A 72-year-old male developed pneumocystis carinii pneumonia stemming from an HIV infection and began HAART at the Department of Hematology, Osaka Medical College, Takatsuki, Japan. Prior to HAART, the patient had shown rapidly progressing retinopathy in the right eye accompanied by vitreous hemorrhage, tractional retinal detachment, and neovascular glaucoma, ultimately leading to early-onset blindness. After starting HAART, the progression of the retinopathy in the left eye became slower compared to the right eye, with corrected visual acuity improving to 0.6 after vitrectomy, despite being accompanied by vitreous hemorrhage. The patient's overall condition has remained stable following the operation, and the condition of the ocular fundus in the left eye has also settled. Conclusion: Significant differences were found in the progres-
\end{abstract}




\section{Case Reports in Ophthalmology}

sion rate of PDR with HIV infection between before and after starting HAART. Our findings suggest that early administration of HAART to HIV patients with diabetic retinopathy is crucial for maintaining visual function.

\section{Introduction}

Patients with a human immunodeficiency virus (HIV) infection commonly exhibit retinal microvasculopathy, such as retinal bleeding or soft exudate [1, 2], and it has been reported that the HIV infection acts as an aggravating factor in cases of diabetic retinopathy (DR) [3, 4]. It has also been reported that when administering highly active anti-retroviral therapy (HAART) to HIV patients, it alleviates DR [5]. In the present study, we report on a patient with proliferative DR (PDR) and HIV infection who exhibited extremely active PDR followed by a rapid onset of blindness in the right eye. The progression of visual disturbance in the left eye was slowed down after starting HAART, and the patient's visual function was rescued after vitrectomy.

\section{Case Report}

We report on a 72-year-old male who had developed diabetes mellitus at 20 years of age, and whose blood glucose levels $(\mathrm{HbA1c}=11 \%)$ had been poorly controlled. The patient's local doctor diagnosed PDR and administered panretinal photocoagulation on both eyes. However, the DR got worse in the patient's right eye, and he was referred to our hospital for vitrectomy. Upon initial examination, the patient's visual acuity (VA) was as follows: $\mathrm{RV}=\left(0.02 \mathrm{x}+0.125 \mathrm{D}=\mathrm{C}-1.5 \mathrm{DA} \times 95^{\circ}\right), \mathrm{LV}=\left(0.8 \mathrm{x}+1.5 \mathrm{D}=\mathrm{C}-1.0 \mathrm{DA} \times 90^{\circ}\right)$. The intraocular pressure was $9 \mathrm{~mm} \mathrm{Hg}$ in the right eye and $10 \mathrm{~mm} \mathrm{Hg}$ in the left eye, and mild cataracts were found in both eyes. In addition, both eyes showed PDR in the ocular fundus, and his right eye exhibited vitreous hemorrhage.

Although the patient was scheduled for vitrectomy on his right eye, we decided to postpone the operation due to the lack of control of the patient's diabetes and to first bring his blood glucose levels under control in our hospital's Department of Internal Medicine. Three weeks after the initial examination, the patient developed a sudden fever, which upon further evaluation was diagnosed as pneumocystis carinii pneumonia (PCP). Blood tests revealed that the patient was HIV positive $(102.90$ s/co under the CLIA method, above $64 \times$ under the PA method), and the illness was diagnosed as PCP associated with AIDS patients. Blood tests revealed anemia (RBC $2.79 / \mu \mathrm{L}, \mathrm{Ht} 8.8 \mathrm{~g} / \mathrm{dL}$ ), blood clotting irregularities (a prothrombin test time of $15.3 \mathrm{~s}$ ), were negative for hepatitis type $\mathrm{B}$ and type $\mathrm{C}$ antibodies, positive for syphilis reaction (positive RPR test, positive TPHA test), positive for CD4, and revealed T lymphocytes $333 / \mu \mathrm{L}$. A subsequent deterioration of the patient's overall condition rendered vitrectomy on his right eye problematic.

Following the patient's first examination at our department, the retinopathy in his right eye devolved rapidly accompanied by vitreous hemorrhage, tractional retinal detachment, and neovascular glaucoma leading to nearly complete loss of vision 3 months later. The patient's VA was then slight light perception in the right eye and 0.7 in the left eye. The patient then started HAART at the Department of Internal Medicine to combat the HIV infection. After starting HAART, the progression of PDR in the patient's left eye clearly slowed down compared to his right eye, stabilizing to a point at which the patient experienced lapses of 


\section{Case Reports in Ophthalmology}

mild vitreous hemorrhage. At 3 months after starting HAART, the patient's VA in the left eye remained at 0.7 , yet had worsened to no light perception in the right eye. However, at 8 months after starting HAART, we found an aggravation of the vitreous hemorrhage and vitreoretinal adhesions (Fig. 1) and performed vitrectomy and cataract surgery on the patient's left eye, as his corrected VA had dropped to 0.09 (noncorrectable). We performed cataract and vitreous surgery to remove the vitreous hemorrhage. The posterior vitreous had not detached, so the fibrovascular proliferative membranes from the optic disc to the vascular arcade were removed using a vitreous cutter (Fig. 2). Subsequently, we performed panretinal endophotocoagulation on the peripheral area. The patient's postoperative course was favorable and he maintains a corrected VA of 0.6 at 2 years postoperatively (Fig. 3). He still has no light perception in his right eye.

\section{Discussion}

According to reports published by the Japan Health, Labor, and Welfare Ministry's AIDS Trends Committee, there has been a yearly rise in the number of HIV and AIDS patients [6]. In light of the increasing numbers of patients with DR as well, it is suspected that there will be a rise in the number of chances to diagnose HIV and AIDS patients with DR [7].

HIV and AIDS patients commonly exhibit retinal microvasculopathy, known as HIV retinopathy. Clinical findings indicate that HIV retinopathy exhibits similar findings as DR, i.e., retinal bleeding and soft exudate, thus making it problematic to differentiate these two diseases. When these findings are observed in the ocular fundus of HIV and AIDS patients with diabetes, it is extremely challenging to determine whether they are caused by pure retinal microvasculopathy, DR, or a combination of the two. Physicians are generally left with no other choice but to make a holistic evaluation based on the diseased organs affected by diabetes, transitions in the patient's HbA1c levels, the number of CD4-positive lymphocytes which are an indicator of the immunological state of HIV-infected patients, and the period until the lymphocytes dissipate.

Jaworski [8] has reported that there are morphological differences in the soft exudates seen in HIV retinopathy and DR, with a larger number of boomerang-shaped soft exudates in HIV retinopathy. Another critical point to distinguish the two is the presence of cytomegalovirus retinitis, which is a common complication in HIV and AIDS patients [9].

Observations of the ocular fundus in the present case did not turn up any characteristic retinal vasculitis caused by cytomegalovirus retinitis, vascular obstruction, or findings of hard exudate, suggesting a lack of complications from cytomegalovirus retinitis. Since HIV retinopathy and DR are both forms of retinal microvasculopathy, it is conceivable that the retinopathy might become more severe in cases in which these two diseases are combined. In fact, past reports have indicated that HIV infection acts as an aggravating factor for retinopathy in cases with DR complications $[10,11]$. On the other hand, it has also been reported that when HAART is used to treat HIV infections, it has an alleviating effect on DR [5].

HAART is a treatment that prevents the onset of AIDS by administering multiple antiHIV 1 drugs in a combination tailored to each patient's individual symptoms and physical constitution, in order to suppress the proliferation of the virus. The life expectancy for HIV and AIDS patients has improved due to the introduction of HAART [12], and a further increase in cases where there are complications with DR is expected as HIV and AIDS patients reach older ages. Shiibashi et al. [6] reported a case in which PDR was quelled with declines in vitreous hemorrhage and changes in exudate upon starting HAART to treat PDR combined 
with HIV infection. In the present case, the patient's overall condition temporarily deteriorated severely due to PCP prior to beginning HAART, and the progress clearly seemed to be more rapid compared to standard PDR. However, after starting HAART, the progression of retinopathy in the patient's left eye clearly slowed relative to the progression in the right eye. Although the patient eventually suffered from vitreous hemorrhage and needed to undergo vitrectomy, the level of difficulty in performing the vitrectomy was relatively low, and the patient has maintained relatively favorable VA following the operation as well. However, it should be noted that protease inhibitors are one of the drugs used in HAART, and they are reported to have side effects in patients with diabetes and high blood pressure [13-15]. Hence, it is important to pay strict attention to whether or not patients undergoing HAART have exhibited symptoms such as retinal vein or artery occlusion aside from DR symptoms.

In conclusion, this patient with an HIV infection and PDR demonstrated significant differences in the progression rate of retinopathy before and after starting HAART. Our findings suggest that an early administration of HAART in HIV patients with DR is possibly crucial for maintaining visual function.

\section{Acknowledgements}

The authors wish to thank John Bush for editing the manuscript.

\section{Statement of Ethics}

This case study was approved by the Ethics Committee of Osaka Medical College.

\section{Disclosure Statement}

There are no conflicts of interest to report for all authors.

\section{References}

1 Nemoto T: HIV/AIDS surveillance and prevention studies in Japan: summary and recommendations. AIDS Educ Prev 2004;16(suppl A):27-42.

-2 Jabs DA, Green WR, Fox R, Polk BF, Bartlett JG: Ocular manifestations of acquired immune deficiency syndrome. Ophthalmology 1989;96:1092-1099.

-3 Wilson R: Cotton wool spots in AIDS: a review. J Am Optom Assoc 1994;65:110-116.

4 Accorinti M, Pirraglia MP, Corradi R, Corsi C, Fabiani C, Pivetti-Pezzi P: Changing patterns of ocular manifestations in HIV seropositive patients treated with HAART. Eur J Ophthalmol 2006;16:728-732. Pivetti-Pezzi P, Accorinti M, Ciapparoni V, Vullo V, Aiuti F: Antiretroviral therapy and HIV-related retinal microangiopathy. AIDS 1997;11:1890-1891.

6 Shiibashi M, Yoshimoto M, Shigeeda T, Fujimura S, Kato S: A case of proliferative diabetic retinopathy that regressed on human immunodeficiency virus treatment. Int J STD AIDS 2015;26:516-517.

-7 Satlin MJ, Hoover DR, Glesby MJ: Glycemic control in HIV-infected patients with diabetes mellitus and rates of meeting American Diabetes Association management guidelines. AIDS Patient Care STDS 2011;25:5-12.

8 Jaworski C: Morphology of the HIV versus the diabetic cotton wool spot. Optom Vis Sci 2000;77:600604.

-9 O’Donnell JJ, Jacobson MA: Cotton-wool spots and cytomegalovirus retinitis in AIDS. Int Ophthalmol Clin 1989;29:105-107.

10 Kaye-Wilson LG, Fleck BW: AIDS and diabetic retinopathy. AIDS 1991;5:902. 


\section{Case Reports in \\ Ophthalmology}

\begin{tabular}{l|l}
\hline DOI: $10.1159 / 000452789$ & (c) 2016 The Author(s). Published by S. Karger AG, Basel \\
\hline
\end{tabular}
www.karger.com/cop

Kitagaki et al: A Case of Proliferative Diabetic Retinopathy with HIV Infection in Which HAART Possibly Influenced the Prognosis of Visual Function

11 Adán A, Goday A, Ferrer J, Cabot J: Diabetic retinopathy associated with acquired immunodeficiency syndrome. Am J Ophthalmol 1990;109:744-745.

12 Palella FJ Jr, Delaney KM, Moorman AC, Loveless MO, Fuhrer J, Satten GA, Aschman DJ, Holmberg SD: Declining morbidity and mortality among patients with advanced human immunodeficiency virus infection. HIV Outpatient Study Investigators. N Engl J Med 1998;338:853-860.

-13 Justman JE, Benning L, Danoff A, Minkoff H, Levine A, Greenblatt RM, Weber K, Piessens E, Robison E, Anastos K: Protease inhibitor use and the incidence of diabetes mellitus in a large cohort of HIV infected women. J Acquir Immune Defic Syndr 2003;32:298-302.

14 Carr A, Samaras K, Thorisdottir A, Kaufmann GR, Chisholm DJ, Cooper DA: Diagnosis, prediction, and natural course of HIV-1 protease-inhibitor-associated lipodystrophy, hyperlipidaemia, and diabetes mellitus: a cohort study. Lancet 1999;353:2093-2099.

15 Heath KV, Hogg RS, Chan KJ, Harris M, Montessori V, O’Shaughnessy MV, Montanera JS: Lipodystrophyassociated morphological, cholesterol and triglyceride abnormalities in a population-based HIV/AIDS treatment database. AIDS 2001;15:231-239.

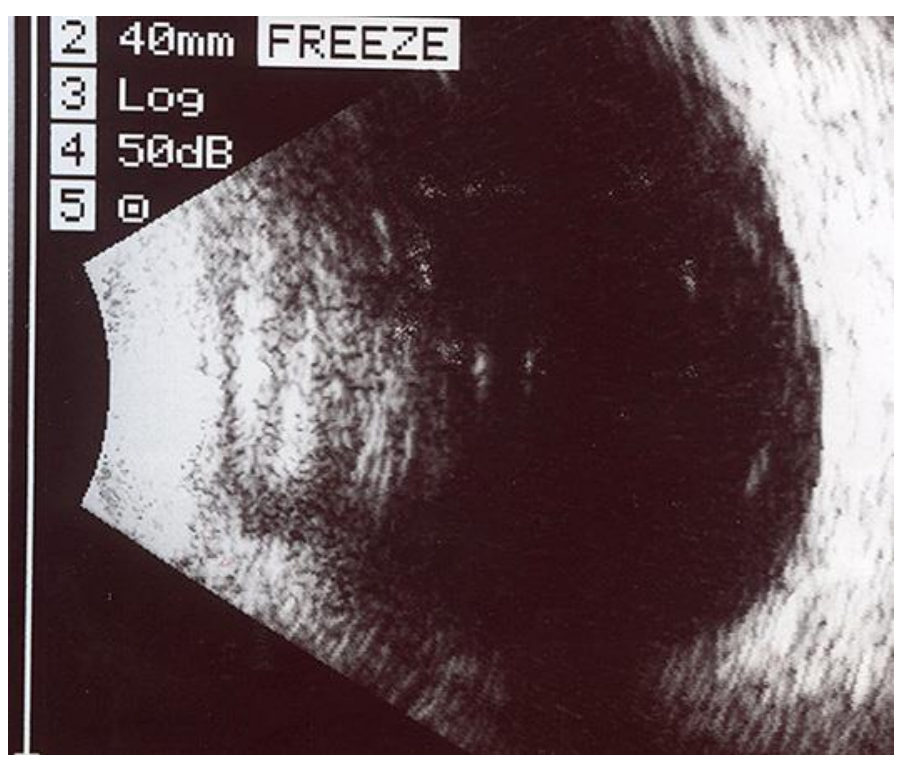

Fig. 1. B-mode ultrasonic tomographic image of the left eye of a patient with proliferative diabetic retinopathy and human immunodeficiency virus infection. Aggravated vitreous hemorrhage and vitreoretinal adhesions can be observed. 


\section{Case Reports in Ophthalmology}

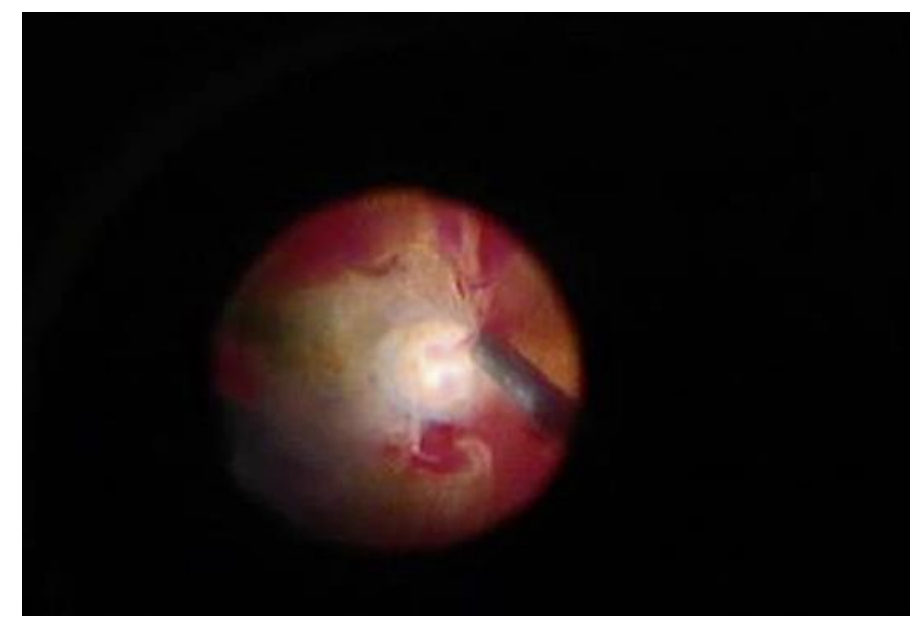

Fig. 2. Intraoperative findings during vitrectomy in the patient' left eye. Fibrovascular proliferative membranes from the optic disc to the vascular arcade were removed using a vitreous cutter.

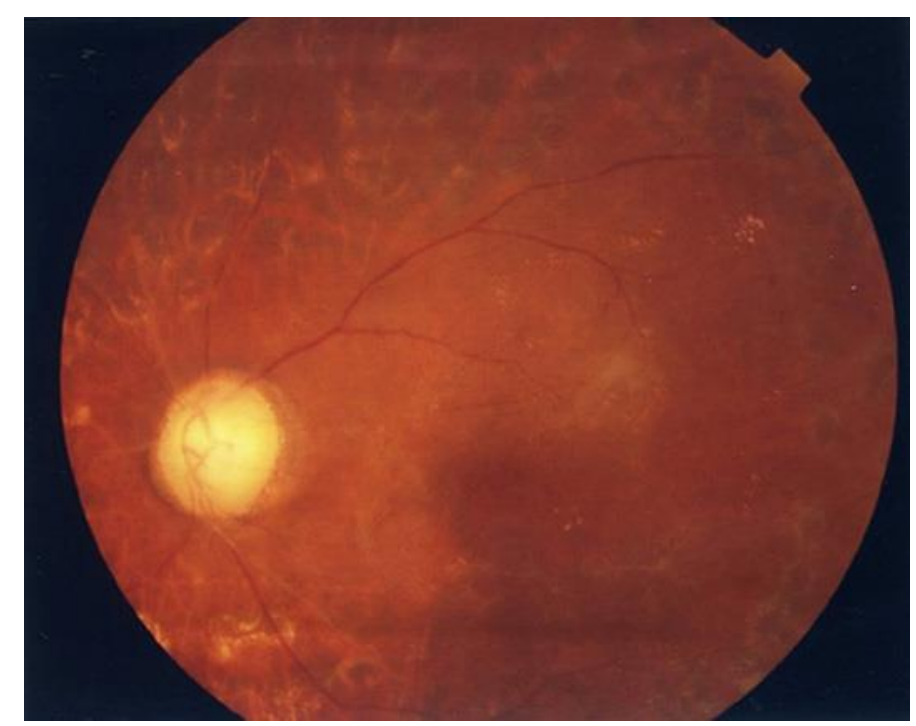

Fig. 3. Postoperative fundus photograph of the patient's left eye. Fundus visualization was recovered, and the patient's visual acuity improved to 0.6. 